\title{
Unrestricted Aggregate Signatures
}

\author{
Mihir Bellare ${ }^{1}$, Chanathip Namprempre ${ }^{2}$, and Gregory Neven ${ }^{3}$ \\ 1 Department of Computer Science \& Engineering \\ University of California San Diego \\ 9500 Gilman Drive, La Jolla, California 92093, USA. \\ Email: mihir@cs.ucsd.edu, URL: http://www-cse.ucsd.edu/users/mihir \\ 2 Electrical Engineering Department, Thammasat University. \\ Rangsit Campus, Klong Luang, Patumtani, Thailand 12121. \\ Email: nchanath@engr.tu.ac.th, URL: http://www.engr.tu.ac.th/ ${ }^{\text {nchanath }}$ \\ 3 Department of Electrical Engineering, Katholieke Universiteit Leuven. \\ Kasteelpark Arenberg 10, B-3001 Heverlee-Leuven, Belgium. \\ Email: Gregory.Neven@esat.kuleuven.be, URL: http://www.neven.org
}

\begin{abstract}
Secure use of the BGLS [7] aggregate signature schemes is restricted to the aggregation of distinct messages (for the basic scheme) or per-signer distinct messages (for the enhanced, prepend-public-key version of the scheme). We argue that these restrictions preclude interesting applications, make usage of the schemes error-prone and are generally undesirable in practice. Via a new analysis and proof, we show how the restrictions can be lifted, yielding the first truly unrestricted aggregate signature scheme. Via another new analysis and proof, we show that the distinct signer restriction on the sequential aggregate signature schemes of [14] can also be dropped, yielding an unrestricted sequential aggregate signature scheme.
\end{abstract}

\section{Introduction}

AgGregate signatures. An aggregate signature (AS) scheme [7] is a digital signature scheme with the additional property that a sequence $\sigma_{1}, \ldots, \sigma_{n}$ of individual signatures - here $\sigma_{i}$ is the signature, under the underlying base signature scheme, of some message $m_{i}$ under some public key $p k_{i}$ - can be condensed into a single, compact aggregate signature $\sigma$ that simultaneously validates the fact that $m_{i}$ has been signed under $p k_{i}$ for all $i=1, \ldots, n$. There is a corresponding aggregate verification process that takes input $\left(p k_{1}, m_{1}\right), \ldots,\left(p k_{n}, m_{n}\right), \sigma$ and accepts or rejects. Aggregation is useful to reduce bandwidth and storage, and is especially attractive for mobile devices like sensors, cell phones, and PDAs where communication is more power-expensive than computation and contributes significantly to reducing battery life.

SchemES. Boneh, Gentry, Lynn, and Shacham [7] present an aggregate signature scheme based on the BLS signature scheme [9]. We call it $\mathcal{A S}$ - 1 and represent it succinctly in the first row of Table 1 . $\mathcal{A S}$-1, however, has some limitations. As the table shows, the aggregate verification process, on inputs $\left(p k_{1}, m_{1}\right), \ldots$, 
$\left(p k_{n}, m_{n}\right), \sigma$, rejects if the messages $m_{1}, \ldots, m_{n}$ are not distinct. The restriction is crucial because, without it, as shown in [7], the scheme is subject to a forgery attack. The consequence, however, is to restrict the ability to aggregate to settings where the messages signed by the signers are all different. BGLS recognize this limitation and suggest a workaround. Specifically, they say: "It is easy to ensure the messages are distinct: The signer simply prepends her public key to every message she signs ..." [7, Section 3.2]. They stop short of specifying a scheme in full, but since it is clearly their intention to "reduce to the previous case," our understanding is that they are advocating the modified scheme in which the signature of a message $m$ under $p k$ is the BLS signature of the enhanced message $M=p k \| m$ under $p k$ while aggregate verification is done by applying the aggregate verification procedure of $\mathcal{A S}-1$ to $\left(p k_{1}, p k_{1} \| m_{1}\right), \ldots,\left(p k_{n}, p k_{n} \| m_{n}\right), \sigma$. However, if so, in this scheme, which we call $\mathcal{A S}$-2, the aggregate verification process will reject unless the enhanced messages $p k_{1}\left\|m_{1}, \ldots, p k_{n}\right\| m_{n}$ are distinct. (Why? Because the aggregate verification process of $\mathcal{A S}-1$ rejects unless the messages are distinct, and the role of the messages is now played by the enhanced messages.) The consequence is that the ability to aggregate is restricted to settings where the enhanced messages signed by the signers are all different. That is, the limitations have been reduced, but not eliminated.

OUR RESULT. We ask whether there exists a truly unrestricted proven-secure aggregate signature scheme. Namely, there should be no distinctness-based restriction of any kind, whether on messages or enhanced messages. We show that the answer is yes. Our result is a new, direct analysis of the security of enhancedmessage signature aggregation which shows that the distinctness condition in the aggregate verification process of $\mathcal{A S}-2$ - namely that this process rejects if any two enhanced messages are the same - can be dropped without compromising security. In other words, an unrestricted scheme can be obtained by the natural adaptation of $\mathcal{A S}-2$ in which the distinctness condition in the verification is simply removed and all else is the same. This scheme, which we denote $\mathcal{A S}$ - 3 , is summarized in the last row of Table 1 . The fact that $\mathcal{A S}$ - 3 is very close to $\mathcal{A S}-2$ is a plus because it means existing implementations can be easily patched.

We clarify that the security of $\mathcal{A S}$ - 3 is not proved in [7]. They prove secure only $\mathcal{A S}-1$. The security of $\mathcal{A S}-2$ is a consequence, but the security of $\mathcal{A S}-3$ is not. What we do instead is to directly analyze security in the case that signatures are on enhanced messages. Our analysis explicitly uses and exploits the presence of the prepended public keys to obtain the stronger conclusion that $\mathcal{A S}-3$ (not just $\mathcal{A S}$-2) is secure.

Motivation. The limitation of $\mathcal{A S}-2$-namely that aggregation is restricted to settings where no two enhanced messages are the same - may seem minor, because all it says is that a set of signatures to be aggregated should not contain duplicates, meaning multiple signatures by a particular signer of a particular message. However, as we now explain, there are several motivations for schemes like $\mathcal{A S}$ - 3 where aggregation is possible even in the presence of duplicates.

Consider a sensor network deployed in a remote area, for example, an environment monitoring network such as the tsunami early warning system already 


\begin{tabular}{|l|l|l|}
\hline Scheme & Sign & Aggregate verification process accepts iff \\
\hline \hline $\mathcal{A S}$-1 $[7]$ & $\mathrm{H}(m)^{x}$ & $\mathbf{e}(\sigma, g)=\prod_{i=1}^{n} \mathbf{e}\left(\mathrm{H}\left(m_{i}\right), g^{x_{i}}\right)$ and $m_{1}, \ldots, m_{n}$ all distinct \\
$\mathcal{A S}$-2 $[7]$ & $\mathrm{H}\left(g^{x} \| m\right)^{x}$ & $\begin{array}{c}\mathbf{e}(\sigma, g)=\prod_{i=1}^{n} \mathbf{e}\left(\mathrm{H}\left(g^{x_{i}} \| m_{i}\right), g^{x_{i}}\right) \text { and } \\
g^{x_{1}}\left\|m_{1}, \ldots, g^{x_{n}}\right\| m_{n} \text { all distinct }\end{array}$ \\
\hline $\mathcal{A S}$-3 & $\mathrm{H}\left(g^{x} \| m\right)^{x}$ & $\mathbf{e}(\sigma, g)=\prod_{i=1}^{n} \mathbf{e}\left(\mathrm{H}\left(g^{x_{i}} \| m_{i}\right), g^{x_{i}}\right)$ \\
\hline
\end{tabular}

Table 1. The aggregate signature schemes we discuss. Here e: $\mathbb{G}_{1} \times \mathbb{G}_{2} \rightarrow \mathbb{G}_{T}$ is a bilinear map, $g$ is a generator of $\mathbb{G}_{2}$ known to all parties, and $H:\{0,1\}^{*} \rightarrow \mathbb{G}_{1}$ is a hash function. The second column shows the signature of a message $m$ under public key $g^{x}$, generated using secret key $x$. In all cases, a sequence of signatures is aggregated by simply multiplying them in $\mathbb{G}_{1}$. The third column shows under what conditions the aggregate verification algorithm accepts $\sigma$ as a valid aggregate signature of messages $m_{1}, \ldots, m_{n}$ under public keys $g^{x_{1}}, \ldots, g^{x_{n}}$ respectively.

in operation in the Indian Ocean [11]. The sensors periodically record measurements from the environment and send them to a monitoring center. In applications where integrity is important (say, to prevent attackers from raising a false alarm that a tsunami is coming), each sensor node must sign its data. The center aggregates these data and the signatures to save storage. (The schemes discussed here permit on-line aggregation in which one can maintain a current aggregate and aggregate into it a received signature.) However, environmental measurements can certainly repeat over time! Indeed, especially in stable conditions, we would expect frequent repeats. Thus, a single signer (sensor) may sign the same data (measurement) many times. In general, whenever the data being signed is drawn from a small space, not just messages, but even enhanced messages can repeat and an unrestricted aggregate signature scheme is necessary.

Perhaps an even more important reason to prefer unrestricted schemes is that they are less likely to be misused or to result in unexpected errors. An application designer contemplating using $\mathcal{A S}$ - 2 must ask herself whether, in her application, enhanced messages might repeat. This may not only be hard to know in advance, but might also change with time. (Experience has repeatedly shown that once a piece of cryptography is deployed, it is used for purposes or applications beyond those originally envisaged.) With an unrestricted scheme, the application designer is freed from the need to worry about whether the setting of her application meets the restrictions, reducing the chance of error. In general, application designers and programmers have a hard enough time as it is to make error-free use of cryptography. Asking them to understand message distinctness restrictions and anticipate whether their application meets them is an added burden and one more place where things can go wrong.

POSSIBLE WORKAROUNDS. Various ways to get around message distinctness restrictions may come to mind, but these workarounds either do not work or tend 
to be more complex or restrictive than direct use of an unrestricted scheme. For example, one could have the verifier drop from its input list $\left(p k_{1}, m_{1}\right), \ldots,\left(p k_{n}\right.$, $\left.m_{n}\right), \sigma$ any pair $p k_{i}, m_{i}$ that occured previously in the list, but then, assuming $\sigma$ was a correct aggregate signature of the given list, it will not be a correct aggregate signature for the truncated list, and verification will fail. Another possibility is that the aggregator refrain from including in the aggregate any signature corresponding to a public key and message whose signature is already in the aggregate. But aggregation may be done on-line, and the aggregator may know only the current aggregate and the incoming signature, and have no way to tell whether or not it is a duplicate. Nonces (timestamps or sequence numbers) could be added to messages to render them unique, but this would complicate the application and increase cost. ${ }^{4}$ Being able to use $\mathcal{A S}$ - 3 without any worries about signature or message replication is simpler, easier, and more practical.

RESULTS FOR SASs. A sequential aggregate signature (SAS) scheme [14] permits a more restrictive kind of aggregation in which the signers must participate in the process and use their secret keys. Imagine the signers forming a chain. In step $i$, the $i$-th signer receives from the previous one a current aggregate, and, using its secret key, it aggregates into this its own signature, passing the new aggregate on to the next signer in the chain. The output of the final signer is the aggregate signature. Although clearly less powerful than general aggregate signature (GAS) schemes - following [8] we now use this term for the BGLS-type aggregate signatures discussed above in order to distinguish them from SASsthe argument of [14] is that sequential aggregation is possible for base signature schemes other than BLS or may be done more cheaply. Specifically, Lysyanskaya, Micali, Reyzin, and Shacham [14] present a SAS scheme based on certified [5] claw-free trapdoor permutations.

However, the model and schemes of [14] also have some limitations. They require that no public key can appear more than once in a chain. That is, the signers who are part of a signing chain must be distinct. But in practice there are many reasons to prefer to allow aggregation even when a particular signer signs multiple messages, meaning when there are loops in the chain and public keys can repeat. Certainly, the previously discussed motivations are still true. Namely, in applications like signing in sensor nets or ad hoc networks, a particular signer (sensor) will be producing many signatures and it would be convenient to allow these to be aggregated. More importantly, an unrestricted SAS scheme is more misuse resistant because it does not require the application designer to try to predict in advance whether or not there will be repeated signers in a prospective chain. But in fact the restrictions in [14] are even greater than the ones for $\mathcal{A S}-2$, for they say not only that one cannot aggregate when a particular signer signs a particular message twice, but that one cannot aggregate even when a particular

\footnotetext{
${ }^{4}$ Nonces might be added anyway to prevent replay attacks. If so, well and good. But replay attacks are not always a concern. In some settings, an adversary might be able to inject bogus data, yet be unable to eavesdrop. Without eavesdropping, the adversary clearly cannot replay data. This could be true for the ocean sensor scenario we discussed above.
} 
signer signs two or more messages that are distinct. This makes the number of excluded applications even larger, for it is common that a particular signer needs to sign multiple messages.

Our result - analogous to the GAS case - is a new analysis and proof that shows that the restrictions imposed by [14] on their schemes can be lifted without impacting security (that is, verification can just drop the condition that one reject if there are repeated public keys, and security is preserved), yielding unrestricted schemes. Again, that only a minor modification to the scheme is needed is convenient if existing implementations need to be updated. The precise result statement and the accompanying proof are in the full version of this paper [3].

Tight Reduction Results. The security of $\mathcal{A S}-1, \mathcal{A S}$ - 2 , and $\mathcal{A S}$ - 3 is based on the coCDH problem. However, none of the security reductions - namely, those of [7] in the first two cases and ours in the last - are tight. By applying a technique of [12], we obtain an alternative scheme $\mathcal{A S}$ - 4 with a tight security reduction to coCDH. When implemented over the same group, $\mathcal{A S}-1, \mathcal{A S}-2, \mathcal{A S}$-3, and $\mathcal{A S}-4$ all have about the same computational cost, the price for $\mathcal{A S}-4$ being a slightly larger aggregate signature. (Specifically, one needs one extra bit per constituent signature.) However, due to the tight reduction, $\mathcal{A S}-4$ is actually more efficient than the other schemes when one compares them at the same (provable) security level. (Because to achieve a given level of security, $\mathcal{A S}-4$ needs a smaller group than the other schemes.) We also obtain an analogous result for the SAS case. The statements and the proofs of our tight reduction results are in the full version of this paper [3].

Related Work. Lu, Ostrovsky, Sahai, Shacham, and Waters [13] present a SAS scheme for which they can lift the distinct signer restriction, as follows. If a signer wishes to add its signature of a message $M_{\text {new }}$ to an aggregate $S$ which already contains its signature $S_{\text {old }}$ on some message $M_{\text {old }}$, then it removes $S_{\text {old }}$ from $S$ and then adds back in a signature on the message $M_{\text {new }} \| M_{\text {old }}$. However, their scheme is weaker than the others we have discussed - ours or those of $[7,14]$ — with regard to some security properties and also with regard to efficiency. Specifically, [13] use the certified public key model $[1,6]$, which reflects the assumption that signers provide strong ZK proofs of knowledge of secret keys to the $\mathrm{CA}$ at key-registration, an assumption that we, following $[7,14]$, do not make. Also, in the scheme of [13], public keys are very large, namely 162 group elements, which is particularly expensive if public keys have to be transmitted along with signatures. In contrast, other schemes have short public keys. On the other hand, the proofs of [13] are in the standard model, while ours, following [7, 14], are in the random oracle model of [4].

Interestingly, in a survey paper, Boneh, Gentry, Lynn, and Shacham [8] present $\mathcal{A S}$-3, claiming that the results of [7] prove it secure. However, this appears to be an oversight because, as we have seen, the results of [7] prove $\mathcal{A S}$-2 secure, not $\mathcal{A S}$-3. By proving $\mathcal{A S}$ - 3 secure via our direct analysis, we are filling the gap and validating the claim of [8]. Shacham's Ph.D thesis [15] notes that the concrete security of the reduction of [7] can be slightly improved by replacing messages with enhanced ones, but he does not claim security of $\mathcal{A S}$ - 3 . 


\section{Notation and Basic Definitions}

Notation And CONVEntions. If $x$ is a string, then $|x|$ is the length of $x$. We denote by $x_{1}\|\cdots\| x_{n}$ an encoding of objects $x_{1}, \ldots, x_{n}$ as a binary string from which the constituent objects are uniquely recoverable. When the objects can be encoded as strings whose length is known from the context, simple concatenation will serve the purpose. If $S$ is a finite set, then $|S|$ is its size, and $s \stackrel{\$}{\leftarrow} S$ means that $s$ is chosen at random from $S$. We let $e$ denote the base of the natural logarithm. An algorithm may be randomized unless otherwise indicated. An adversary is an algorithm. If $A$ is an algorithm then $y \stackrel{\$}{\leftarrow} A\left(x_{1}, x_{2}, \ldots\right)$ means that $y$ is the result of executing $A$ on fresh random coins and inputs $x_{1}, x_{2}, \ldots$ We denote by $\left[A\left(x_{1}, x_{2}, \ldots\right)\right]$ the set of all possible outputs of $A$ on the indicated inputs, meaning the set of all strings that have a positive probability of being output by $A$ on inputs $x_{1}, x_{2}, \ldots$. We let $\operatorname{Maps}(D)$ denote the set of all functions with domain $\{0,1\}^{*}$ and range $D$.

Digital SignATURE SCHEMEs. We recall definitions for (standard) signature schemes [10] in the random-oracle ( $\mathrm{RO})$ model [4]. A signature scheme $\mathcal{D S}=(\mathrm{Kg}$, Sign, Vf) is specified by three algorithms, the last deterministic. Via $(p k, s k) \stackrel{\$}{\leftarrow}$ $\mathrm{Kg}$, a signer generates its public key $p k$ and matching secret key $s k$, where $\mathrm{H}:\{0,1\}^{*} \rightarrow D$ is a random oracle whose range $D$ is a parameter of the scheme. Via $\sigma \stackrel{\$}{\leftarrow} \operatorname{Sign}^{\mathrm{H}}(s k, m)$ the signer can generate a signature $\sigma$ on a message $m$. A verifier can run $\mathrm{Vf}^{\mathrm{H}}(p k, m, \sigma)$ to obtain a bit, with 1 indicating accept and 0 reject. The consistency (or correctness) condition is that the probability that $\mathrm{Vf}^{\mathrm{H}}(p k, m, \sigma)=1$ must equal one for all messages $m \in\{0,1\}^{*}$, where the probability is over the following experiment:

$$
(p k, s k) \stackrel{\$}{\leftarrow} \mathrm{Kg} ; \mathrm{H} \stackrel{\$}{\leftarrow} \operatorname{Maps}(D) ; \sigma \stackrel{\$}{\leftarrow} \operatorname{Sign}^{\mathrm{H}}(s k, m) .
$$

To capture security (unforgeability under chosen-message attack), we define the advantage of an adversary B, denoted by $\mathbf{A d v}_{\mathcal{D S}}^{\text {uf-cma }}(\mathrm{B})$, as the probability that $\mathrm{Vf}^{\mathrm{H}}(p k, m, \sigma)=1$, where the probability is over the following experiment:

$$
(p k, s k) \stackrel{\$}{\leftarrow} \mathrm{Kg} ; \mathrm{H} \stackrel{\$}{\longleftarrow} \operatorname{Maps}(D) ;(m, \sigma) \stackrel{\$}{\longleftarrow} \mathrm{B}^{\operatorname{Sign}^{\mathrm{H}}(s k, \cdot), \mathrm{H}} .
$$

To make this meaningful, we only consider adversaries that are legitimate in the sense that they never queried the message in their output to their sign oracle. We say that $\mathcal{D S}$ is $\left(t, q_{\mathrm{S}}, q_{\mathrm{H}}, \epsilon\right)$-secure if no adversary B running in time at most $t$, invoking the signature oracle at most $q_{\mathrm{S}}$ times and the random oracle at most $q_{\mathrm{H}}$ times, has advantage more than $\epsilon$.

\section{$3 \quad$ Unrestricted General Aggregate Signatures}

GAS sCHEMES. A general aggregate signature (GAS) scheme [7] $\mathcal{A S}=(\mathrm{Kg}$, Sign, Agg, AVf) consists of four algorithms, the last deterministic. The key generation and signing algorithms are exactly as for standard digital signatures. Via 
$\sigma \stackrel{\$}{\leftarrow} \operatorname{Agg}^{\mathrm{H}}\left(\left(p k_{1}, m_{1}, \sigma_{1}\right), \ldots,\left(p k_{n}, m_{n}, \sigma_{n}\right)\right)$, anyone can aggregate a sequence of public key, message, and signature triples to yield an aggregate signature $\sigma$. A verifier can run $\mathrm{AVf}^{\mathrm{H}}\left(\left(p k_{1}, m_{1}\right), \ldots,\left(p k_{n}, m_{n}\right), \sigma\right)$ to obtain a bit, with 1 indicating accept and 0 reject.

SECURITY. The security requirement of [7] is strong, namely that an adversary find it computationally infeasible to produce an aggregate forgery involving an honest signer, even when it can play the role of all other signers, in particular choosing their public keys, and can mount a chosen-message attack on the honest signer. To capture this, we define the advantage of an adversary $A$ as

$$
\operatorname{Adv}_{\mathcal{A S}}^{\text {agg-uf }}(\mathrm{A})=\operatorname{Pr}\left[\operatorname{AVf}^{\mathrm{H}}\left(\left(p k_{1}, m_{1}\right), \ldots,\left(p k_{n}, m_{n}\right), \sigma\right)=1\right]
$$

where the probability is over the experiment

$$
\begin{aligned}
& (p k, s k) \stackrel{\$}{\leftarrow} \mathrm{Kg} ; \mathrm{H} \stackrel{\$}{\leftarrow} \operatorname{Maps}(D) ; \\
& \left(\left(p k_{1}, m_{1}\right), \ldots,\left(p k_{n}, m_{n}\right), \sigma\right) \stackrel{\$}{\longleftarrow} \mathrm{A}^{\operatorname{Sign}^{\mathrm{H}}(s k, \cdot), \mathrm{H}}(p k) .
\end{aligned}
$$

To make this meaningful, we only consider adversaries that are legitimate in the sense that there must exist $i \in\{1, \ldots, n\}$ such that $p k_{i}=p k$ but $A$ never queried $m_{i}$ to its signing oracle. Thus, the honest signer here is the one whose keys are $p k, s k$, and we are asking that the aggregate forgery include some message and signature corresponding to this honest signer, but the adversary never legitimately obtained a signature of this message. We say that $\mathrm{A}\left(t, q_{\mathrm{S}}, n_{\max }, q_{\mathrm{H}}, \epsilon\right)$ breaks $\mathcal{A S}$ if it runs in time at most $t$, invokes the signature oracle at most $q_{\mathrm{S}}$ times, invokes the hash oracle at most $q_{\mathrm{H}}$ times, outputs a forgery containing at most $n_{\max }$ public-key-message pairs, and has advantage strictly greater than $\epsilon$. We say that $\mathcal{A S}$ is $\left(t, q_{\mathrm{S}}, n_{\max }, q_{\mathrm{H}}, \epsilon\right)$-secure if there is no adversary that $\left(t, q_{\mathrm{S}}, n_{\mathrm{max}}, q_{\mathrm{H}}, \epsilon\right)$-breaks $\mathcal{A S}$.

A significant feature of this definition, highlighted in [7], is that A can choose $p k_{1}, \ldots, p k_{n}$ as it wishes, in particular as a function of $p k$. Unlike $[1,6,13]$, there is no requirement that the adversary "know" the secret key corresponding to a public key it produces, and this makes the system more practical since it avoids the need for strong zero-knowledge proofs of knowledge [2] of secret keys done to the CA during key-registration. Our results continue to achieve this strong notion of security.

BilineAR mAPS AND COCDH. Let $\mathbb{G}_{1}, \mathbb{G}_{2}, \mathbb{G}_{T}$ be groups, all of the same prime order $p$. Let e: $\mathbb{G}_{1} \times \mathbb{G}_{2} \rightarrow \mathbb{G}_{T}$ be a non-degenerate, efficiently computable bilinear map, also called a pairing. Let $g$ be a generator of $\mathbb{G}_{2}$. For the rest of the paper, we regard $\mathbb{G}_{1}, \mathbb{G}_{2}, \mathbb{G}_{T}, \mathbf{e}, g$ as fixed, globally known parameters, and also let $t_{\exp }$ denote the time to perform an exponentiation in $\mathbb{G}_{1}$. Note that following $[9,7]$ we use the asymmetric setting $\left(\mathbb{G}_{1}, \mathbb{G}_{2}\right.$ are not necessarily equal) and must also assume there exists an isomorphism $\psi: \mathbb{G}_{2} \rightarrow \mathbb{G}_{1}$. (The first is in order to make signatures as short as possible, and the second is required for the security proofs.) We define the advantage of an adversary $A$ in solving the 
coCDH problem as

$$
\mathbf{A d v}^{\mathrm{co-cdh}}(\mathrm{A})=\operatorname{Pr}\left[\mathrm{A}\left(g, g^{a}, h\right)=h^{a}: h \stackrel{\&}{\leftarrow} \mathbb{G}_{1} ; a \stackrel{\$}{\leftarrow} \mathbb{Z}_{p}\right] .
$$

We say that the coCDH problem is $\left(t^{\prime}, \epsilon^{\prime}\right)$-hard if no algorithm $\mathrm{A}$ running in time at most $t^{\prime}$ has advantage strictly more than $\epsilon^{\prime}$ in solving it. Note that when $\mathbb{G}_{1}=\mathbb{G}_{2}$, the coCDH problem becomes the standard CDH problem in $\mathbb{G}_{1}$, whence the name.

The $\mathcal{B} L \mathcal{S}$ sCheme. We recall the $\mathcal{B} \mathcal{S}$ standard signature scheme of [9]. The signer chooses a secret key $x \stackrel{\&}{\leftarrow} \mathbb{Z}_{p}$ and computes the corresponding public key $X \leftarrow g^{x}$. Let $\mathrm{H}:\{0,1\}^{*} \rightarrow \mathbb{G}_{1}$ be a random oracle. The signature of message $m$ is $\sigma=\mathrm{H}(m)^{x}$, which can be verified by checking that $\mathbf{e}(\sigma, g)=\mathbf{e}(\mathrm{H}(m), X)$. Regarding security, we have the following:

Theorem 1 [9] If the coCDH problem is $\left(t^{\prime}, \epsilon^{\prime}\right)$-hard, then the $\mathcal{B L S}$ standard signature scheme is $\left(t, q_{\mathrm{S}}, q_{\mathrm{H}}, \epsilon\right)$-secure for any $t, q_{\mathrm{S}}, q_{\mathrm{H}}, \epsilon$ satisfying $\epsilon \geq e\left(q_{\mathrm{S}}+\right.$ 1) $\cdot \epsilon^{\prime}$ and $t \leq t^{\prime}-t_{\exp }\left(q_{\mathrm{H}}+2 q_{\mathrm{S}}\right)$.

The GAS SChEMES We CONSIDER. We consider four closely related aggregate signature schemes that we denote $\mathcal{A S}$ - $0, \mathcal{A S}$ - $1, \mathcal{A S}$ - $2, \mathcal{A S}$-3. These schemes share common key generation and aggregation algorithms, but differ in their signing and verification algorithms. All use a random oracle $H:\{0,1\}^{*} \rightarrow \mathbb{G}_{1}$. Key generation is exactly as in the $\mathcal{B} \mathcal{L} S$ scheme: the secret key is an exponent $x \stackrel{\&}{\leftarrow} \mathbb{Z}_{p}$ and the corresponding public key is $X=g^{x}$. For $\mathcal{A S}-0$ and $\mathcal{A S}-1$, the signing algorithm is the $\mathcal{B L S}$ one, namely the signature on message $m$ is $\sigma=\mathrm{H}(m)^{x}$. For $\mathcal{A S}-2$ and $\mathcal{A S}$-3, a signature on $m$ under public key $X$ is $\sigma=\mathrm{H}(X \| m)^{x}$. For all schemes, aggregation is done by simply multiplying the signatures, i.e. $\sigma=\prod_{i=1}^{n} \sigma_{i}$ in $\mathbb{G}_{1}$. Verification is different for each scheme. On inputs $\left(X_{1}, m_{1}\right), \ldots,\left(X_{n}, m_{n}\right), \sigma$, the verification algorithm of $\mathcal{A S S} \boldsymbol{O}$ accepts iff $\mathbf{e}(\sigma, g)=\prod_{i=1}^{n} \mathbf{e}\left(\mathrm{H}\left(m_{i}\right), X_{i}\right)$. The verification algorithms of the other schemes are depicted in Table 1 . In particular, $\mathcal{A S}$ - 1 rejects if $m_{1}, \ldots, m_{n}$ are not all distinct, $\mathcal{A S}$ - 2 rejects if $X_{1}\left\|m_{1}, \ldots, X_{n}\right\| m_{n}$ are not all distinct, while $\mathcal{A S}$-3 performs no such checks.

Consistency CONDitions. The consistency condition (under what conditions correctly generated aggregates are accepted by the verifier) differs from scheme to scheme, and is in fact the place where the restrictions they make surface in a formal sense. $\mathcal{A S}$ - 0 and $\mathcal{A S}$ - 3 meet the natural, strongest possible consistency requirement, namely that

$$
\operatorname{Pr}\left[\operatorname{AVf}^{\mathrm{H}}\left(\left(p k_{1}, m_{1}\right), \ldots,\left(p k_{n}, m_{n}\right), \sigma\right)=1\right]=1
$$

for all positive integers $n$, all messages $m_{1}, \ldots, m_{n} \in\{0,1\}^{*}$ and all $\left(p k_{1}\right.$, $\left.s k_{1}\right), \ldots,\left(p k_{n}, s k_{n}\right) \in[\mathrm{Kg}]$, where the probability is over the experiment $\mathrm{H} \stackrel{\mathbb{R}}{\leftarrow}$ $\operatorname{Maps}(D) ; \sigma_{1} \stackrel{\&}{\leftarrow} \operatorname{Sign}^{\mathrm{H}}\left(s k_{1}, m_{1}\right) ; \cdots ; \sigma_{n} \stackrel{\&}{\leftarrow} \operatorname{Sign}^{\mathrm{H}}\left(s k_{n}, m_{n}\right) ; \sigma \stackrel{\&}{\leftarrow} \operatorname{Agg}^{\mathrm{H}}\left(\left(p k_{1}, m_{1}\right.\right.$, 
$\left.\left.\sigma_{1}\right), \ldots,\left(p k_{n}, m_{n}, \sigma_{n}\right)\right)$. However, $\mathcal{A S}$ - 1 meets this condition only when $m_{1}, \ldots$, $m_{n}$ are distinct and $\mathcal{A S}$ - 2 when $p k_{1}\left\|m_{1}, \ldots, p k_{n}\right\| m_{n}$ are distinct.

Discussion OF SECURITY. An attack provided in [7] shows that $\mathcal{A S}-\boldsymbol{O}$ is insecure. In this attack, however, the forgery output by the adversary contains repeated messages. To exclude the attack, [7] defines $\mathcal{A S}$-1, where the aggregate verification process rejects when messages repeat. They are able to show that this suffices to guarantee security, meaning that they prove $\mathcal{A S}$ - 1 is secure if the coCDH problem is hard. This is their main result. Then they suggest to alleviate the message-distinctness restriction of $\mathcal{A S}-1$ by having each signer prepend its public key to the message before signing. However, they appear to want to argue the security of the resulting aggregate signature scheme as a corollary of their main result on the security of $\mathcal{A S}-1$. If so, verification still needs to check that $X_{1}\left\|m_{1}, \ldots, X_{n}\right\| m_{n}$ are all distinct (otherwise, the result about $\mathcal{A S}$ - 1 does not apply), leading to the $\mathcal{A S}-2$ scheme.

As we have discussed, however, for practical reasons, $\mathcal{A S}-3$ is a preferable scheme. But the results of [7] do not prove it secure. Here is an example that helps to see what the problem is. Suppose there was an adversary A that, on input $p k=X$ and without making oracle query $m$, produced a forgery of the form $(X, m),\left(X^{\prime}, m^{\prime}\right),\left(X^{\prime}, m^{\prime}\right), \sigma$, for some $m^{\prime} \neq m$ and $X^{\prime} \neq X$, that was accepted by the verification procedure of $\mathcal{A S}$-3. Since the output of A contains repeated enhanced messages, the results of [7] do not allow us to rule out the existence of A. Yet, showing that $\mathcal{A S}$-3 meets the notion of security that we have defined does require ruling out the existence of such an $A$.

Theorem 2 If the coCDH problem is $\left(t^{\prime}, \epsilon^{\prime}\right)$-hard, then the $\mathcal{A S}$-3 aggregate signature scheme is $\left(t, q_{\mathrm{S}}, n_{\max }, q_{\mathrm{H}}, \epsilon\right)$-secure for any $t, q_{\mathrm{S}}, n_{\max }, q_{\mathrm{H}}, \epsilon$ satisfying $\epsilon \geq$ $e\left(q_{\mathrm{S}}+1\right) \cdot \epsilon^{\prime}$ and $t \leq t^{\prime}-t_{\exp }\left(2 q_{\mathrm{H}}+2 q_{\mathrm{S}}+3 n_{\max }+1\right)$. I

Our approach to the proof is different from the one used by [7] to prove that $\mathcal{A S}$ - 1 is secure if coCDH is hard. They gave a direct reduction to coCDH, meaning, given an adversary attacking $\mathcal{A S}-1$ they construct and analyze an adversary attacking coCDH. But, in so doing, they end up duplicating a lot of the proof of the security of the $\mathcal{B} L \mathcal{S}$ scheme as given in [9]. Instead, we reduce the security of $\mathcal{A S}$-3 to the security of $\mathcal{B L S}$. That is, we prove the following:

Lemma 3 If the $\mathcal{B L S}$ standard signature scheme is $\left(t^{\prime}, q_{\mathrm{S}}^{\prime}, q_{\mathrm{H}}^{\prime}, \epsilon^{\prime}\right)$-secure then the $\mathcal{A S}-3$ aggregate signature scheme is $\left(t, q_{\mathrm{S}}, n_{\max }, q_{\mathrm{H}}, \epsilon\right)$-secure for any $t, q_{\mathrm{S}}, n_{\max }$, $q_{\mathrm{H}}, \epsilon$ satisfying $\epsilon \geq \epsilon^{\prime}, q_{\mathrm{S}} \leq q_{\mathrm{S}}^{\prime}-n_{\max }, q_{\mathrm{H}} \leq q_{\mathrm{H}}^{\prime}$ and $t \leq t^{\prime}-t_{\mathrm{exp}} \cdot\left(q_{\mathrm{H}}+n_{\max }+1\right)$.

Theorem 2 follows easily from Lemma 3 and Theorem 1. Our modular approach yields a simple proof even though we obtain a somewhat stronger result.

An interesting element of the proof of Lemma 3 is that it involves reducing the security of one random oracle model scheme to another one. Given a forger A against the $\mathcal{A S}-3$ that queries a random oracle, we must build a forger $\mathrm{B}$ against $\mathcal{B L S}$. But $B$ is itself given a random oracle. The idea is that $B$ will answer some of A's queries via its own random oracle and directly simulate the others. 
Subroutine H-SIM $(M)$

If $\left(\exists m: M=X^{*} \| m\right)$ then return $\mathrm{H}_{\mathcal{B L S}}(M)$

Subroutine $\operatorname{SigN-SIM}(m)$

Else If $\mathrm{HT}[M]=\perp$ then $\mathrm{y}[M] \stackrel{\$}{\leftarrow} \mathbb{Z}_{p} ; \operatorname{HT}[M] \leftarrow \psi(g)^{\mathrm{y}[M]}$

$\operatorname{Return}^{\operatorname{Sign}_{\mathcal{B L S}}}\left(x^{*}, X^{*} \| m\right)$

Return $\mathrm{HT}[M]$

Fig. 1. The subroutines for $B$ to simulate the random oracle $H_{A_{\mathcal{S}}-3}(\cdot)$ and the sign oracle $\operatorname{Sign}_{\mathcal{A S}-3}\left(x^{*}, \cdot\right)$. Above, HT and y are associative arrays assumed initially to have value $\perp$ everywhere.

Proof (Lemma 3). Given a forger A that $\left(t, q_{\mathrm{S}}, n_{\max }, q_{\mathrm{H}}, \epsilon\right)$-breaks $\mathcal{A S}$-3, consider the following forger $B$ against the $\mathcal{B} \mathcal{S}$ standard signature scheme. $B$ is given public key $X^{*}=g^{x^{*}}$ as input, and has access to a random oracle $\mathrm{H}_{\mathcal{B L S}}(\cdot)$ and a signing oracle $\operatorname{Sign}_{\mathcal{B L S}}\left(x^{*}, \cdot\right)=\mathrm{H}_{\mathcal{B L S}}(\cdot)^{x^{*}}$. It runs $\mathrm{A}$ on input $X^{*}$ and responds to its $\mathrm{H}_{\mathcal{A S}-3}(\cdot)$ and $\operatorname{Sign}_{\mathcal{A S}-3}\left(x^{*}, \cdot\right)$ oracle queries using the subroutines in Fig. 1.

When A submits a query $M$ to its random oracle $\mathrm{H}_{\mathcal{A S}-3}(\cdot)$, the forger $\mathrm{B}$ executes H-SIM $(M)$. We note here that in some cases the subroutine H-SIM can in turn submit queries to B's random oracle $\mathrm{H}_{\mathcal{B} \mathcal{L S}}(\cdot)$. When $\mathrm{A}$ submits a query $m$ to its sign oracle $\operatorname{Sign}_{\mathcal{A S}-3}\left(x^{*}, \cdot\right)$, the forger B executes $\operatorname{SigN-SIM}(m)$. Eventually, A halts and outputs a forgery $\left(X_{1}, m_{1}\right), \ldots,\left(X_{n}, m_{n}\right), \sigma$. Since A is legitimate, we know that there exists $i \in\{1, \ldots, n\}$ such that $X_{i}=X^{*}$ and $m_{i}$ has never been queried to $\operatorname{Sign}_{\mathcal{A S}-3}\left(x^{*}, \cdot\right)$. We let $i^{*}$ denote the smallest integer $i$ for which this happens. Now B defines the sets:

$$
\begin{aligned}
I & =\left\{i \mid X_{i}=X^{*} \text { and } m_{i}=m_{i^{*}}\right\} \\
J & =\left\{i \mid X_{i}=X^{*} \text { and } m_{i} \neq m_{i^{*}}\right\} \\
K & =\left\{i \mid X_{i} \neq X^{*}\right\} .
\end{aligned}
$$

Note that $I$ is non-empty since $i^{*} \in I$. Clearly, we have that $I \cup J \cup K=\{1, \ldots, n\}$ and that $I, J, K$ are disjoint. Now, we can assume without loss of generality that $n<p$, because otherwise $\mathrm{B}$ can trivially forge and output a $\mathcal{B} \mathcal{L S}$ signature under $X^{*}$ in time $O\left(n t_{\exp }\right)$ via exhaustive search for $x^{*}$. This means that $|I| \in \mathbb{Z}_{p}^{*}$, and hence has an inverse modulo $p$ that we denote by $l$. Now, for each $i \in J$, our adversary B executes $\operatorname{Sign-SIM}\left(m_{i}\right)$ to obtain $\sigma_{i} \leftarrow \operatorname{Sign}_{\mathcal{B L S}}\left(x^{*}, X^{*} \| m_{i}\right)$. For each $i \in K$, it calls its subroutine $\mathrm{H}-\operatorname{SIM}\left(X_{i} \| m_{i}\right)$, thereby ensuring that $\mathrm{y}\left[X_{i} \| m_{i}\right]$ is defined, lets $y_{i} \leftarrow \mathrm{y}\left[X_{i} \| m_{i}\right]$, and then lets $\sigma_{i} \leftarrow \psi\left(X_{i}\right)^{y_{i}}$, which we note is the $\mathcal{B L S}$ signature of $X_{i} \| m_{i}$ under public key $X_{i}$. Finally, B lets

$$
M^{*} \leftarrow X^{*} \| m_{i^{*}} \quad \text { and } \quad \sigma^{*} \leftarrow\left(\sigma \cdot \prod_{i \in J \cup K} \sigma_{i}^{-1}\right)^{l},
$$

and outputs $\left(M^{*}, \sigma^{*}\right)$ as its forgery.

For the analysis, we first argue that if A's forgery is valid then B's forgery is valid too. Assuming the former, the verification equation of $\mathcal{A S}$ - 3 tells us that

$$
\begin{aligned}
\mathbf{e}(\sigma, g) & =\prod_{i=1}^{n} \mathbf{e}\left(\mathrm{H}_{\mathcal{A S}-3}\left(X_{i} \| m_{i}\right), X_{i}\right) \\
& =\prod_{i \in I} \mathbf{e}\left(\mathrm{H}_{\mathcal{B L S}}\left(X^{*} \| m_{i^{*}}\right), X^{*}\right) \cdot \prod_{i \in J} \mathbf{e}\left(\mathrm{H}_{\mathcal{B L S}}\left(X^{*} \| m_{i}\right), X^{*}\right)
\end{aligned}
$$




$$
\begin{aligned}
& \prod_{i \in K} \mathbf{e}\left(\mathrm{H}_{\mathcal{A S}-3}\left(X_{i} \| m_{i}\right), X_{i}\right) \\
= & \mathbf{e}\left(\mathrm{H}_{\mathcal{B L S}}\left(X^{*} \| m_{i^{*}}\right), X^{*}\right)^{|I|} \cdot \prod_{i \in J \cup K} \mathbf{e}\left(\sigma_{i}, g\right) .
\end{aligned}
$$

Above, (2) is true because $\sigma_{i}$, as computed above by $\mathrm{B}$, is the $\mathcal{B L S}$ signature of $X_{i} \| m_{i}$ under public key $X_{i}$, for all $i \in J \cup K$. We then applied the verification equation of the $\mathcal{B} \mathcal{S S}$ scheme. Now we see that if $\sigma^{*}$ is defined by (1) then, from the above and the fact that $|I| \cdot l \equiv 1(\bmod p)$ we have

$$
\begin{aligned}
\mathbf{e}\left(\sigma^{*}, g\right) & =\mathbf{e}(\sigma, g)^{l} \cdot \prod_{i \in J \cup K} \mathbf{e}\left(\sigma_{i}, g\right)^{-l} \\
& =\mathbf{e}\left(\mathrm{H}_{\mathcal{B L S}}\left(X^{*} \| m_{i^{*}}\right), X^{*}\right)^{|I| \cdot l \bmod p} \cdot \prod_{i \in J \cup K} \mathbf{e}\left(\sigma_{i}, g\right)^{l} \cdot \prod_{i \in J \cup K} \mathbf{e}\left(\sigma_{i}, g\right)^{-l} \\
& =\mathbf{e}\left(\mathrm{H}_{\mathcal{B L S}}\left(M^{*}\right), X^{*}\right),
\end{aligned}
$$

which means that $\sigma^{*}$ is a valid $\mathcal{B} \mathcal{L S}$ signature of $M^{*}$ under public key $X^{*}$.

Furthermore, it is easy to see that the answers that B provided to the oracle queries of $A$ are distributed identically to the ones that $A$ would have obtained from its oracles in the game defining its advantage. The last thing we need to check is that $\mathrm{B}$ is legitimate, meaning did not query $M^{*}=X^{*} \| m_{i^{*}}$ to its $\operatorname{Sign}_{\mathcal{B L S}}\left(x^{*}, \cdot\right)$ oracle. But it did not do so while answering $\operatorname{Sign}_{\mathcal{H S}-3}\left(x^{*}, \cdot\right)$ oracle queries of A because A, being legitimate itself, did not query $m_{i^{*}}$ to its $\operatorname{Sign}_{\mathfrak{A S}-3}\left(x^{*}, \cdot\right)$ oracle. Now B also called $\operatorname{Sign}_{\mathcal{B L S}}\left(x^{*}, \cdot\right)$ on $X^{*} \| m_{i}$ for all $i \in J$, but by definition of $J$, we know that $m_{i} \neq m_{i^{*}}$. Putting everything together, we get $\mathbf{A d v}_{\mathcal{B L S}}^{\text {uf-cma }}(\mathrm{B}) \geq \mathbf{A d v}_{\mathcal{A S}-3}^{\text {agg-uf }}(\mathrm{A})$.

Finally, we analyze the resource usage of B. For $q_{\mathrm{S}}$, we note that B makes sign queries in only two situations: (1) whenever $A$ makes a sign query, so does $\mathrm{B}$, and (2) once $\mathrm{A}$ outputs a forgery, $\mathrm{B}$ makes $|J| \leq n_{\max }$ additional sign queries. For $q_{\mathrm{H}}$, it is easy to see that $\mathrm{B}$ makes at most the same number of random oracle queries to $\mathrm{H}_{\mathcal{B L S}}$ as $\mathrm{A}$ makes to $\mathrm{H}_{\mathcal{A S}-3}$. For $t$, notice that $\mathrm{B}$ (1) answers $q_{\mathrm{H}}$ random oracle queries, each of which results in a call to H-SIM, (2) possibly makes $n_{\max }$ more calls to $\mathrm{H}$-SIM after A outputs its forgery, and (3) computes one exponentiation in the last step to convert A's forgery into its own. Thus, the claimed running time bound follows, and the proof is complete.

\section{Acknowledgments}

Mihir Bellare was supported by NSF grants CNS-0524765, CNS-0627779, and a gift from Intel Corporation. Chanathip Namprempre was supported by the Thailand Research Fund. Gregory Neven is a Postdoctoral Fellow of the Research Foundation Flanders, and was supported in part by the Concerted Research Action Ambiorics 2005/11 of the Flemish Government and the European Commission through the IST Programme under Contract IST-2002-507932 ECRYPT. We thank the ICALP 2007 anonymous referees for their valuable comments. 


\section{References}

1. M. Bellare, A. Boldyreva, and J. Staddon. Randomness re-use in multi-recipient encryption schemes. In Y. Desmedt, editor, PKC 2003, volume 2567 of LNCS, pages 85-99. Springer-Verlag, Jan. 2003.

2. M. Bellare and O. Goldreich. On defining proofs of knowledge. In E. F. Brickell, editor, CRYPTO'92, volume 740 of LNCS, pages 390-420. Springer-Verlag, Aug. 1992.

3. M. Bellare, C. Namprempre, and G. Neven. Unrestricted aggregate signatures. Full version of this paper. Available from http://eprint.iacr.org/2006/285.

4. M. Bellare and P. Rogaway. Random oracles are practical: A paradigm for designing efficient protocols. In ACM CCS 93, pages 62-73. ACM Press, Nov. 1993.

5. M. Bellare and M. Yung. Certifying permutations: Noninteractive zero-knowledge based on any trapdoor permutation. Journal of Cryptology, 9(3):149-166, 1996.

6. A. Boldyreva. Threshold signatures, multisignatures and blind signatures based on the gap-Diffie-Hellman-group signature scheme. In Y. Desmedt, editor, PKC 2003, volume 2567 of $L N C S$, pages 31-46. Springer-Verlag, Jan. 2003.

7. D. Boneh, C. Gentry, B. Lynn, and H. Shacham. Aggregate and verifiably encrypted signatures from bilinear maps. In E. Biham, editor, EUROCRYPT 2003, volume 2656 of $L N C S$, pages 416-432. Springer-Verlag, May 2003.

8. D. Boneh, C. Gentry, B. Lynn, and H. Shacham. A survey of two signature aggregation techniques. RSA's CryptoBytes, 6(2), Summer 2003.

9. D. Boneh, B. Lynn, and H. Shacham. Short signatures from the Weil pairing. In C. Boyd, editor, ASIACRYPT 2001, volume 2248 of LNCS, pages 514-532. Springer-Verlag, Dec. 2001.

10. S. Goldwasser, S. Micali, and R. L. Rivest. A digital signature scheme secure against adaptive chosen-message attacks. SIAM Journal on Computing, 17(2):281308, Apr. 1988.

11. Intergovernmental Oceanographic Commission of UNESCO. Towards the establishment of a tsunami warning and mitigation system for the Indian Ocean. Available at http://ioc3.unesco.org/indotsunami/. Last accessed April 13, 2007.

12. J. Katz and N. Wáng. Efficiency improvements for signature schemes with tight security reductions. In ACM CCS 03, pages 155-164. ACM Press, Oct. 2003.

13. S. Lu, R. Ostrovsky, A. Sahai, H. Shacham, and B. Waters. Sequential aggregate signatures and multisignatures without random oracles. In S. Vaudenay, editor, EUROCRYPT 2006, volume 4004 of LNCS. Springer-Verlag, May 2006.

14. A. Lysyanskaya, S. Micali, L. Reyzin, and H. Shacham. Sequential aggregate signatures from trapdoor permutations. In C. Cachin and J. Camenisch, editors, EUROCRYPT 2004, volume 3027 of LNCS, pages 74-90. Springer-Verlag, May 2004.

15. H. Shacham. New Paradigms in Signature Schemes. PhD thesis, Stanford University, 2005. 